\title{
Could Information Energy resolve Hubble Tension?
}

\author{
Michael Paul Gough \\ University of Sussex, Brighton, BN1 9QT, UK.: m.p.gough@sussex.ac.uk
}

\begin{abstract}
Stellar heated gas and dust has a universe total entropy/information content of $\sim 10^{86}$ bits. At typical temperatures $\sim 10^{7}$ the equivalent $N \mathrm{k}_{B} T \ln (2)$ information energy $\sim 10^{70} \mathrm{~J}$ is comparable to the $m c^{2}$ of the universe's $\sim 10^{53} \mathrm{~kg}$ of baryons. At low red-shifts, $z<1.35$ this dark energy contribution provides a near constant energy density, with an equation of state parameter, $w=-1.03 \pm 0.05$, effectively emulating a cosmological constant to within $1.8 \%$ in Hubble parameter, $H(a)$. Earlier, $z>1.35$, the information energy contribution was phantom, $w=-1.82 \pm 0.08$. This dark energy differs from the cosmological constant, $\Lambda$, by $\Delta w_{0}=-0.03 \pm 0.05$ and $\Delta w_{a}=-0.79 \pm 0.08$, sufficient to account for the value of the 'Hubble Tension' between early and late universe $H_{0}$ values. An information energy model will fit most observations as well as $\Lambda$, and also resolve Hubble tension and cosmological coincidence problems. Furthermore, information energy could also account for many effects previously attributed to dark matter.
\end{abstract}

Keywords: Dark Energy; $\Lambda$ CDM model; Cosmological Constant; Hubble Tension.

\section{Introduction}

The standard $\Lambda \mathrm{CDM}$ model has successfully accounted for many features of today's universe. However, as measurements improve in accuracy, a significant difference, or tension, has been found between the early and the late universe values for the Hubble constant, $H_{0}$.

Planck measurements of the Cosmic Microwave Background, $\mathrm{CMB}$, originating from red-shifts, $\mathrm{z} \sim 1100$, provide a $\Lambda \mathrm{CDM}$ model dependent value for today's Hubble Constant, $H_{0}$, of $67.4 \pm 0.5 \mathrm{kms}^{-1} \mathrm{Mpc}^{-1}$ [1]. Recent independent CMB measurements by the Atacama Cosmology Telescope [2] support Planck with a value, $H_{0}=67.9 \pm 1.5 \mathrm{kms}^{-1} \mathrm{Mpc}^{-1}$. This is also consistent with values derived from Baryon Acoustic Oscillations [3].

In contrast to these early universe measurements, $H_{0}$ measured in the late universe by a variety of techniques yields values closer to $74 \mathrm{kms}^{-1} \mathrm{Mpc}^{-1}$. 'Standard candles' provided by type 1a supernovae and Cepheid variable stars, provide distance ladders that yield a value $H_{0}=74.03 \pm 1.42 \mathrm{kms}^{-1} \mathrm{Mpc}^{-1}[4,5]$. Other methods have been devised to be independent of any 'standard candle'. For example, time delay measurements of multiple imaged quasars due to strong gravitational lensing [6] provide $H_{0}=73.3+1.7 /-1.8 \mathrm{kms}^{-1} \mathrm{Mpc}^{-1}$, a value differing by 5.36 from the above early universe values. Also the size of edge-on galaxy discs have been determined by the geometry of water maser action occurring in those discs [7] providing $\mathrm{H}_{0}=73.9 \pm 3 \mathrm{kms}^{-1} \mathrm{Mpc}^{-1}$, a value greater than the early universe value at a $95-99 \%$ level of confidence. In contrast to most late universe values, one method using the tip of red giant evolution just before the helium flash as a 
standard distance candle [8] initially yielded a value of $69.8 \pm 1.9 \mathrm{kms}^{-1} \mathrm{Mpc}^{-1}$, intermediate between the early and late universe values. When corrected for the effects of local stellar densities, this value has been shown to increase to $H_{0}$ $=72.4 \pm 2.0 \mathrm{kms}^{-1} \mathrm{Mpc}^{-1}$, in line with other late universe measurements [9].

Initially it was thought that the 'Hubble Tension' might be attributed to systematic errors [10], but over the last couple of years the late universe measurements have become ever more precise and consistent. The persisting $H_{0}$ tension implies a problem or tension with the $\Lambda$ CDM model, possibly even leading to new physics beyond $\Lambda \mathrm{CDM}[4,11]$. Suggested causes of the tension include: a time-dependent dark energy instead of the cosmological constant, $\Lambda$; a universe with non-zero curvature; a dark matter interaction; an early dark energy; and additional relativistic particles $[4,11]$.

In this paper we consider the specific case of one particular time-dependent source of dark energy and show that it can account for the Hubble tension.

\section{Information Energy as a source of Dark Energy.}

Landauer's Principle provides an equivalent energy for each bit of information or bit of entropy. Landauer showed that information is physical, since the erasure of a bit of information in a system at temperature, $T$, results in the release of a minimum $\mathrm{k}_{B} T \ln (2)$ of energy to the system's surroundings [12,13]. Landauer's principle has now been experimentally verified for both classical bits and quantum qubits [14-17]. Table 1. lists for various astrophysical phenomena their estimated information bit numbers, typical temperatures, equivalent information energy total, and information energy as a fraction of total baryon $m c^{2}$ [18-22].

\begin{tabular}{|c|c|c|c|c|}
\hline & $\begin{array}{c}\text { Information, } \\
N, \text { Bits }\end{array}$ & $\begin{array}{c}\text { Temperature } \\
T,{ }^{\circ} \mathrm{K} \\
\end{array}$ & $\begin{array}{l}\text { Information energy } \\
N k_{B} T \ln 2, \quad \text { Joules }\end{array}$ & $\begin{array}{c}\text { Information Energy/ } \\
\text { baryon } \mathrm{mc}^{2}\end{array}$ \\
\hline $\begin{array}{c}\text { Stellar heated } \\
\text { gas and dust }\end{array}$ & $\sim 10^{86}$ & $10^{6}-10^{8}$ & $10^{69}-10^{71}$ & $10^{-1}-10^{+1}$ \\
\hline $10^{22}$ stars & $10^{79}-10^{81}$ & $\sim 10^{7}$ & $10^{63}-10^{65}$ & $10^{-7}-10^{-5}$ \\
\hline $\begin{array}{c}\text { Stellar black } \\
\text { holes }\end{array}$ & $10^{97}-6 \times 10^{97}$ & $\sim 10^{-7}$ & $10^{67}-6 \times 10^{67}$ & $10^{-3}-6 \times 10^{-3}$ \\
\hline $\begin{array}{l}\text { Super massive } \\
\text { black holes }\end{array}$ & $\begin{array}{c}10^{102}- \\
3 \times 10^{104}\end{array}$ & $\sim 10^{-14}$ & $10^{65}-3 \times 10^{67}$ & $10^{-5}-3 \times 10^{-3}$ \\
\hline Cold dark matter & $\sim 2 \times 10^{88}$ & $<10^{2} ?$ & $<10^{67}$ & $<10^{-3}$ \\
\hline CMB photons & $\begin{array}{c}10^{88}- \\
2 \times 10^{89}\end{array}$ & 2.7 & $3 \times 10^{65}-6 \times 10^{66}$ & $3 \times 10^{-5}-6 \times 10^{-4}$ \\
\hline Relic neutrinos & $\begin{array}{c}10^{88}- \\
5 \times 10^{89}\end{array}$ & 2 & $2 \times 10^{65}-10^{67}$ & $2 \times 10^{-5}-10^{-3}$ \\
\hline Relic gravitons & $\begin{array}{l}10^{86}- \\
6 \times 10^{87}\end{array}$ & $\begin{array}{l}\sim ? \\
\sim\end{array}$ & $10^{63}-6 \times 10^{64}$ & $10^{-7}-6 \times 10^{-6}$ \\
\hline
\end{tabular}

Table 1. Present information contents, temperatures, and energy contributions

Although some contributions possess higher bit numbers, stellar heated gas and dust clearly makes the dominant information energy contribution at present. The $N \sim 10^{86}$ bits at typical gas and dust temperatures, $T \sim 10^{6}-10^{8}$ have equivalent total $N \mathrm{k}_{B} T \ln (2)$ energies of $10^{69}-10^{71} \mathrm{~J}$, directly comparable to the $\sim 10^{70} \mathrm{~J}$ equivalent $m c^{2}$ energy of the universe's $\sim 10^{53}$ 
$\mathrm{kg}$ baryons. Stellar heated gas and dust information equivalent energy should then be included alongside $m c^{2}$ equivalence of matter in universe energy accounting. Information energy from stellar heated gas and dust could account for today's dark energy density, within an order of magnitude, using accepted physics, relying solely on the experimentally proven Landauer's Principle, combined with realistic entropy estimates, and without invoking new physics.

\section{Dynamic Information Energy: time history.}

In order to include the information energy of stellar heated gas and dust in the universe energy density equation, we need to describe its variation over time by identifying how total bit number, $N(a)$, and typical temperature, $T(a)$, vary as a function of universe scale size, $a$, related to redshift, $z$, by $a=1 /(1+z)$.

Firstly, we assume that, within any sufficiently large volume, the average temperature, $T$, representative of the stellar heated gas and dust varies in proportion to the fraction $f(a)$ of baryons that have formed stars up to that scale size. We can determine the history of $f(a)$ by plotting in Figure 1 a survey of measured stellar mass densities per co-moving volume as a function of scale size, $a$. The filled symbols [23-39] correspond to data compiled for a recent survey of stellar formation measurements (Table 2 of [40]). A subset of these data was already included in previous information energy studies [20-22], and open symbols [41-52] correspond to other measurements used in that previous work but not included in

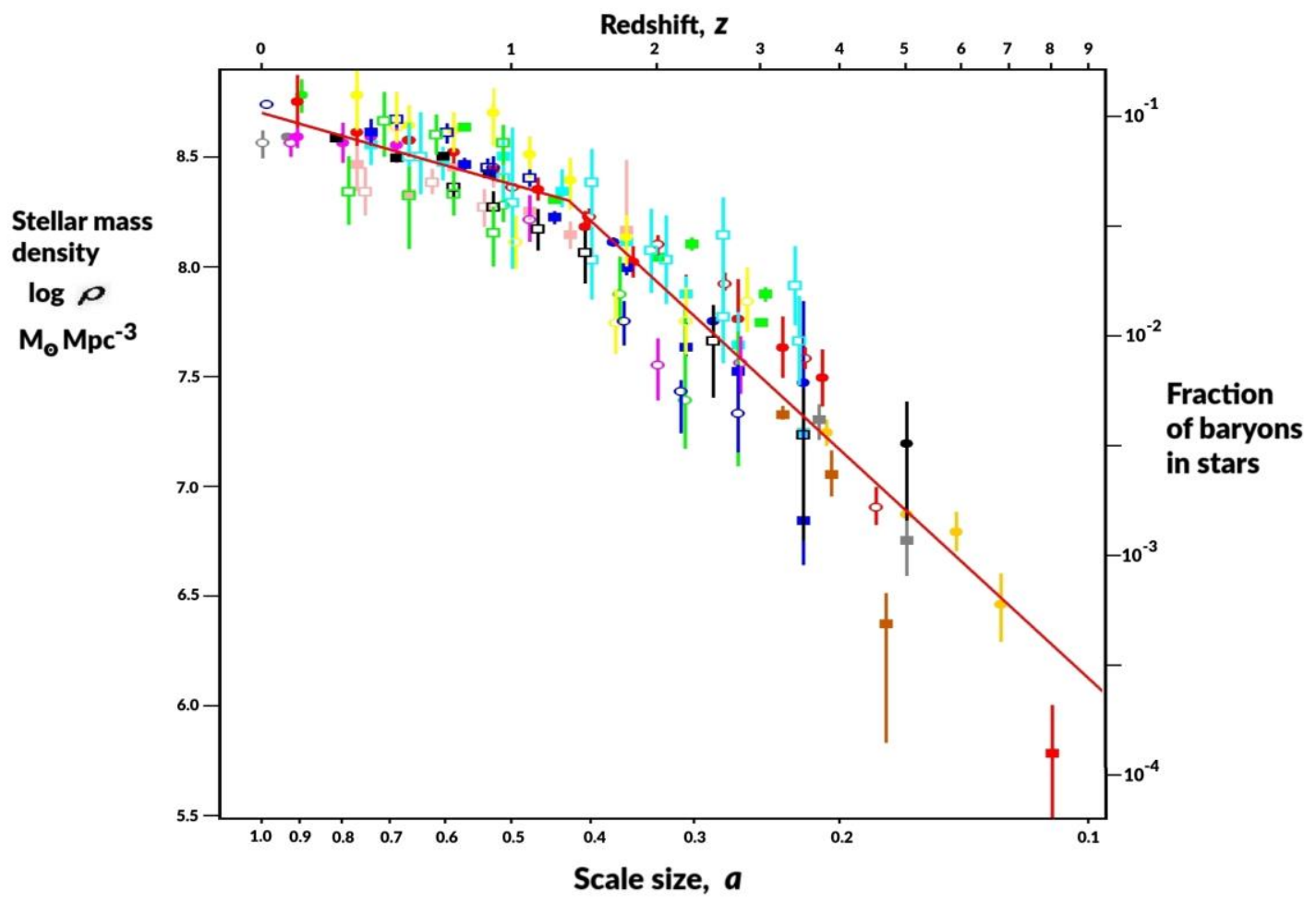

the survey of [40].

Figure 1. Review of stellar mass density measurements for co-moving volumes as a function of universe scale size, $a$. Plotted red lines are power law fits: $a^{+1.08 \pm 0.16}$, for $z<1.35$; and $a^{+3.46 \pm 0.23}$, for $z>1.35$. Source references: Filled symbols: grey circle[23]; dark green circle[24]; magenta circle[25]; pink square[26]; red circle[27]; cyan square[28]; blue square[29]; yellow circle[30]; black square[31]; green square[32]; blue circle[33]; dark green square[34]; brown square[35]; orange circle[36]; grey square[37]; black circle[38]; red square[39]. Open 
symbols: grey circle[41]; dark green circle[42]; magenta circle[43]; pink square[44]; red circle[45]; cyan square[46]; blue square[47]; yellow circle[48]; black square[49]; green square[50]; blue circle[51]; dark green square[52].

In Figure 1 there is a significant change around red-shift, $z \sim 1.35$ from a steep gradient in the past to a weaker gradient in recent times. Fitting straight line power laws (red lines) to data points either side of $z=1.35$, we find power law fits of $a^{+1.08 \pm 0.16}$ for $z<1.35$, and $a^{+3.46 \pm 0.23}$, for $z>1.35$. Then we assume the average stellar heated gas and dust baryon temperature, $T$, proportional to the fraction of baryons in stars $f(a)$, also varied as $a^{+1.08 \pm 0.16}$ for $z<1.35$, and $a^{+3.46 \pm 0.23}$, for $z>1.35$. Measurements of changes in mean galactic electron temperatures over the range $0<z<1$ [53] show a similar temperature time variation as Figure 1, supporting our use of stellar mass densities as a proxy for the gas and dust temperature time variation.

We consider two possibilities for the time variation of total stellar heated gas and dust bit number, $N(a)$. In the first case we assume $N(a)$ simply varies directly proportional to volume as $a^{3}$. In the second case we assume that the total bit number of any large comoving volume is governed by the Holographic Principle [54-56], and varies with the volume's bounding area as $a^{2}$. While the Holographic Principle is generally accepted for black holes at the holographic bound, the holographic bound of the universe is $\sim 10^{123}$ bits and the general principle remains only a conjecture for universal application to cases below that bound [56].

In order to compare the time variation of these information energy models against that of the cosmological constant, we use Weinberg's [57] version of the Friedmann equation [58] for the Hubble parameter, $H(a)$. This equation expresses $H(a)$ in terms of its present value, the Hubble constant, $H_{0}$, and dimensionless energy density parameters, $\Omega$, expressed as a fraction of today's total energy density. Assuming the curvature term is zero, and the radiation term has for some time been negligible compared to the other terms, the $\Lambda \mathrm{CDM}$ model is described simply by equation (1).

$\Lambda \mathrm{CDM}: \quad\left(H(a) / H_{0}\right)^{2}=\Omega_{T o t} a^{-3}+\Omega_{\Lambda}$

where Tot is all matter (dark+baryons) and $\Lambda$, the cosmological constant.

Total information equivalent energy, given by $N \mathrm{k}_{B} T \ln (2)$, is proportional to both $N(a)$ and $T(a)$, and thus proportional to $a^{3} f(a)$ in the volume model, and proportional to $a^{2} f(a)$ in the holographic model, corresponding to information energy density terms $\Omega_{I E}$ $(f(a) / f(1))$ and $\Omega_{I E}(f(a) / f(1)) a^{-1}$, respectively. Then, if the cosmological constant was negligible and information energy provided the sole source of dark energy, we obtain:

$$
\begin{array}{ll}
\text { Information-Volume model: } & \left(H(a) / H_{0}\right)^{2}=\Omega_{\text {Tot }} a^{-3}+\Omega_{I E}(f(a) / f(1)) \\
\text { Information -Holographic model: } & \left(H(a) / H_{0}\right)^{2}=\Omega_{T o t} a^{-3}+\Omega_{I E}(f(a) / f(1)) a^{-1}
\end{array}
$$

In Figure 2 we compare the effects of these two models for an information energy source of dark energy against the cosmological constant using the $\Lambda$ CDM values, setting $\Omega_{T o t}=0.32$ and $\Omega_{\Lambda}=\Omega_{I E}=0.68$ and applying the gradient fits of Figure 1 for $f(a)$.

The difference between the cosmological constant and the two information energy models can only be measured through $H(a)$, dependent on total energy densities. We can 
see from Figure 2, upper plot, that total energy density of the holographic model (red dashes) and the cosmological constant (black dashes) nearly coincide, while that for the volume model (blue dashes) clearly predicts significantly different total energy densities.

Figure 2, lower plot, emphasizes the differences by plotting the percentage difference in expected Hubble parameter for the information energy models relative to that of the cosmological constant model. The volume model differs significantly from the cosmological constant, peaking at $7 \%$ around $a=0.67$. Such a difference at $z=0.5$ from that expected for a cosmological constant should have been easily observed directly by existing expansion measurements, and for this reason we hereafter concentrate on the holographic model. The holographic model difference is less than $1 \%$ for $a>0.4$ and peaks at only $1.8 \%$ around $a=0.33$. This low value is only marginally detectable, even with the next generation of instruments. For example, the ESA Euclid science requirement [59] is to measure $H(a)$ down to an accuracy of $1-2 \%$ in the range $0.33<a<0.67$.
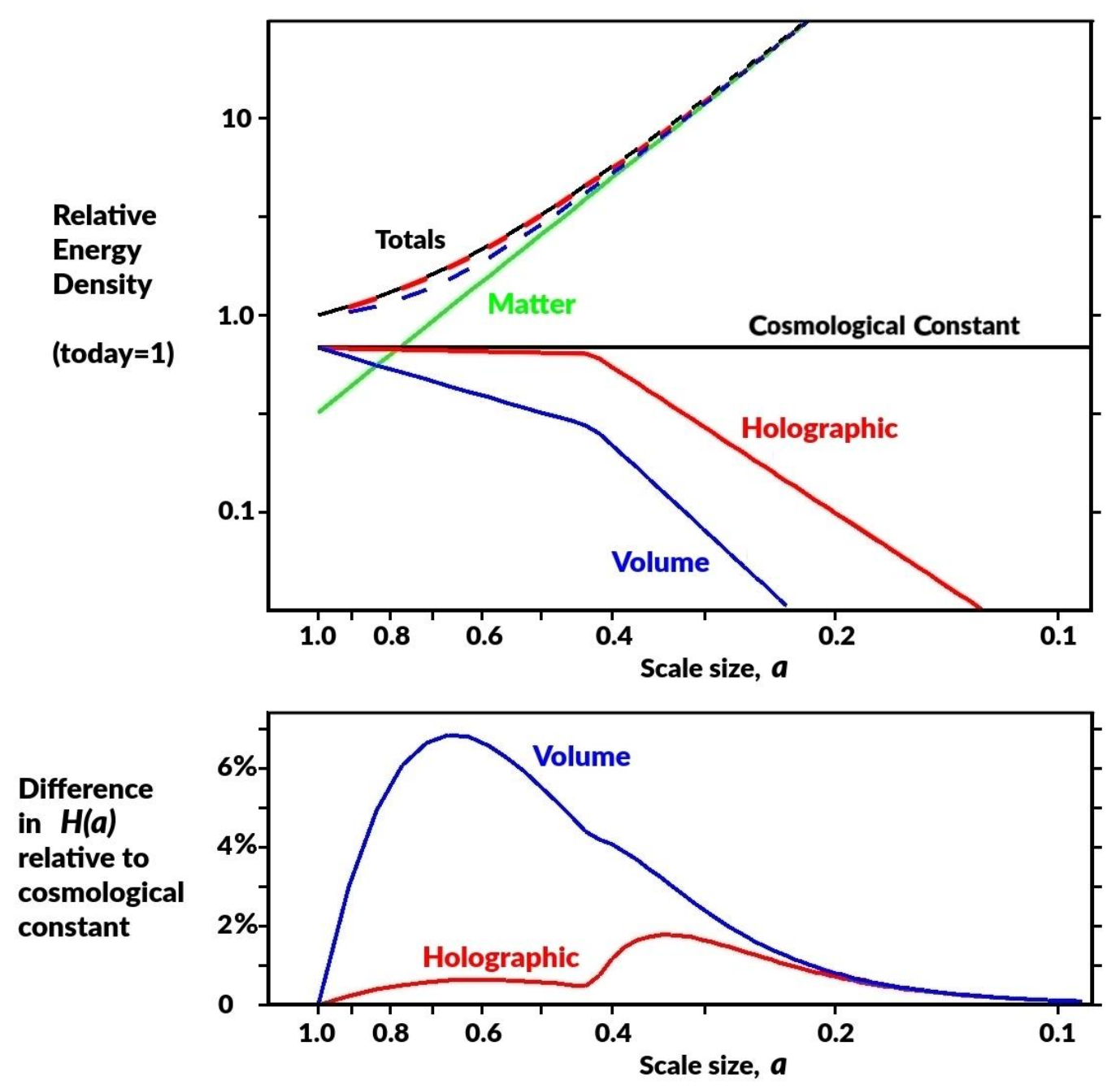

Figure 2. Upper Plot. Energy densities relative to total today $(=1.0)$ for cosmological constant, information energy, all matter, with totals (dashed lines) for all matter+cosmological constant, and all matter+information energy for volume and holographic models. Lower Plot. Difference in Hubble parameter, $H(a)$ to be expected for an information energy source of dark energy relative to that due to a cosmological constant.

Recently, $z<1.35$, total stellar heated gas and dust information energy ( $\alpha N T$ ) has varied as $a^{+3.08 \pm 0.16}$, corresponding to a near constant energy density, or an equation of state 
parameter, value $w=-1.03 \pm 0.05$. In comparison, total information energy in the earlier period, $z>1.35$, varied as $a^{+5.46 \pm 0.23}$, corresponding to a phantom energy with $w=-1.82 \pm 0.08$. Note that Figure 1 uses three times as many stellar mass density study results, including several more recent studies, than was used in the previous work on holographic dark information energy [20-22]. Then information energy of stellar heated gas and dust in the recent period has the characteristics of dark energy, since $f(a)$ closely follows the $a^{+1}$ gradient that would lead to a near constant information energy density to emulate a cosmological constant. Thus information energy can provide a quantitative account of dark energy, accounting for both the present energy density value, $\sim 10^{70} \mathrm{~J}$, and the recent period of near constant energy density.

\section{Earlier Phantom Information Energy can account for Hubble Tension.}

Results of experiments to measure the dark energy equation of state, $w$, often assume a simple shape for the $w(a)$ timeline, using a minimum number of parameters. Most astrophysical datasets, including Planck data [1, 60-62], have been analysed to deduce cosmological parameters using the 'CPL' description [63]: $w(\mathrm{a})=w_{\mathrm{o}}+(1-\mathrm{a}) w_{\mathrm{a}}$. This assumes a smooth variation of $w(a)$ from $w_{0}+w_{\mathrm{a}}$ at very early times, a $<<1$, through to $w_{\mathrm{o}}$ today $(\mathrm{a}=1)$.

The 2013, 2015, and 2018 Planck data releases,[1, 60-62], include several dataset combinations where Planck data have been combined with other types of measurement and analysed using the CPL parameters. Although resultant likelihood regions of $w_{0}-w_{\text {a }}$ space (Fig. 36 of [60], Fig. 28 of [61], Fig. 30 of [1]) include the cosmological constant ( $w_{0}=-1$, $\left.w_{\mathrm{a}}=0\right)$, consistent with $\Lambda \mathrm{CDM}$, there is a clear overall bias towards a phantom dark energy with a significant negative range in $w_{\mathrm{a}}$. Information energy equation of state parameter values, $w=-1.03 \pm 0.05 \mathrm{z}<1.35$, and $w=-1.82 \pm 0.08 z>1.35$, correspond to CPL parameters, $w_{0}=-1.03 \pm 0.05, w_{\mathrm{a}}=-0.79 \pm 0.08$, located close to the centre of these maximum likelihood regions in $w_{0}-w_{\text {a }}$ space. Note these CPL parameters fit the information $w$ values today and very early, but information energy exhibits a much sharper transition at $z \sim 1.35$ than can be fully described by CPL. The difference between the information energy parameters and the parameters for $\Lambda$ is then given by $\triangle w_{0}=-0.03 \pm 0.05$ and $\triangle w_{a}=-0.79 \pm 0.08$.

There is a mounting body of evidence to suggest that the dark energy density is dynamic, with a phantom dark energy $(w(a)<0)$ at earlier times, compatible with an information energy source. Planck CMB data is not strongly constrained without combining with other data sets. On its own this Planck data yields $w=-1.54,+0.62 /-0.50$ corresponding to a $\sim 20$ shift into the phantom regime [60], effectively averaging over the whole range $0<z<1100$. Various combinations of other measurements have found a similar 2-3o shift into the phantom regime [64-68].

While the volume model would lead to easily identifiable differences from $\Lambda \mathrm{CDM}$ at low $\mathrm{z}$, the holographic model emulates a cosmological constant at low $\mathrm{z}$ and, for most phenomena, would be indistinguishable from $\Lambda$ CDM. Identification of the holographic model then requires a comparison between the early and the late universe. We noted above that holographic information energy differs from $\Lambda$ by a difference in CPL parameters of $\Delta w_{0}=-0.03 \pm 0.05$ and $\Delta w_{a}=-0.79 \pm 0.08$. These parameter differences are significant as they closely match the differences required to account for the Hubble tension by such a dynamic dark energy, namely $\Delta w_{0}=-0.08$ and $\Delta w_{a}=-0.8$ (from Figure 4 of [4]). Then holographic information dark energy can quantitatively account for the difference between early and late universe $H_{0}$ measurements, and thus resolve the Hubble Tension problem.

There are other aspects that support this information energy source of dark energy, and explanation for Hubble Tension. The advent of accelerating expansion has been associated 
$[69,70]$ with directly causing a general reduction in galaxy merging, and a reduction in formation rate of structure and stars. This change is evident in Figure 1 in the clear change in stellar mass density gradient at $\mathrm{z} \sim 1.35$ from $a^{+3.46 \pm 0.23}$ to $a^{+1.08 \pm 0.16}$. Assuming dark energy is information energy, once information energy was strong enough to initiate acceleration, this in turn slowed star formation and the growth of information energy itself. The resulting $\sim a^{+1}$ gradient that we observe in Figure 1 is significant as it is the natural limiting value for such feedback. Moreover, for this feedback to operate, information energy would need to be the major, or even sole, source of dark energy. An information energy explanation for dark energy then allows the cosmological constant to take the zero value. A zero value is considered more likely [71], as theoretical estimates for the natural value of $\Lambda$ differ by an enormous factor, $\sim 10^{123}$, from the observed dark energy density.

Star formation needed to have advanced sufficiently for information energy to be strong enough to initiate accelerating expansion. Increasing star formation was also required for the likelihood of intelligent beings evolving to observe this acceleration. Therefore it may not be such a coincidence that we are around when dark energy has a similar order of energy density to that of matter, possibly solving the "why now?" cosmological coincidence problem.

As information energy depends on star and structure formation, it should also be spatially dynamic. This aspect of an information dark energy source might explain recent measurements [72] of apparent directional anisotropies in the value of the Hubble constant.

\section{Algorithmic Information Theory supports dark information energy.}

Algorithmic information theory provides a simple, independent, supporting argument for an information related connection between star formation and accelerating expansion. The algorithmic information content of a system is given by the length of the shortest string of bits that can describe the position and momentum of all particles in the system [73]. This length is often also called the algorithmic complexity or Kolmogorov complexity. Algorithmic information provides insights into the physics, as it has some features in common with thermodynamic entropy, including the requirement to adhere to the $2^{\text {nd }}$ law.

Stars form from clumps in molecular clouds, and especially from clumps in giant molecular clouds. In order to see how star formation has affected the universe algorithmic information content, we can simply consider changes in the universe averaged number of bits required per baryon per dimensional parameter. We must first decide on the level of resolution, or graining, required. For the purposes of our argument, we consider the two limiting resolutions: Planck length $\left(1.6 \times 10^{-35} \mathrm{~m}\right)$, the smallest dimension with any physical meaning; and Fermi length $\left(10^{-15} \mathrm{~m}\right)$, typical of nucleon dimensions and thus the largest to still describe particle interactions. Intergalactic baryons, bounded by the universe $\left(\sim 10^{27} \mathrm{~m}\right)$ require $\sim 205$ bits/parameter at Planck resolution, since the required accuracy is one part in $6 \times 10^{61}$ or one part in $2^{205}$. Similarly, baryons constrained within giant molecular clouds $\left(\sim 10^{18} \mathrm{~m}\right)$ require $\sim 175 \mathrm{bits} /$ parameter, and baryons in a typical star (e.g. the sun $\left.10^{9} \mathrm{~m}\right)$ require $\sim 145$ bits/parameter. At Fermi resolution the three equivalent values are each 65.7 bits/parameter less.

We assume a simple model based on the growth in the measured fraction of baryons that have formed stars up to the present value of $10 \%$ of all baryons (Figure 1). While the bits/parameter of the intergalactic baryons increase by one bit per universe doubling in size, those baryons within giant molecular clouds lose 30 bits per parameter when they form stars. Then Figure 3. shows the resulting average bits/parameter/baryon using the 
growth history of star formation given by the survey of Figure 1 (red lines). For comparison Figure 3. also shows the variation if there was no star formation. The 30 bits lost by the present $10 \%$ of baryons that have formed stars has made a contribution of 3 bits to the average while, between $a=\sim 0.25$ and the present $a=1.0$, the remaining $90 \%$ of baryons have contributed +2 bits to the average. The resulting overall average fall of $\sim 1$ bit/parameter/baryon is clearly independent of resolution between the limits of Planck and Fermi lengths, and implies a significant decrease in the total algorithmic information content of the universe, contrary to the $2^{\text {nd }}$ law.

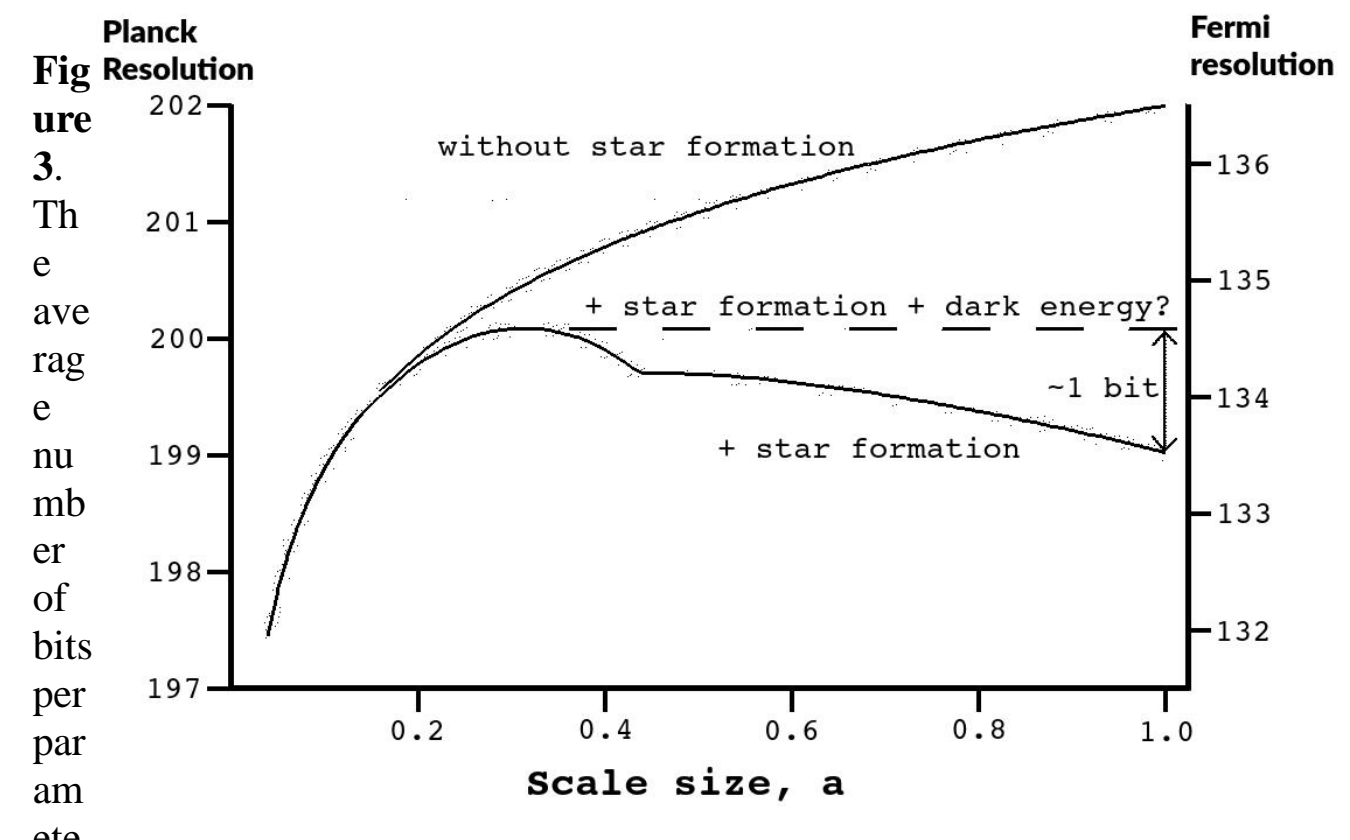

$r$ per baryon, averaged over all universe baryons, needed for an algorithmic information description.

Fortunately, dark energy has additionally increased the total universe energy density by $\sim x 4$, resulting in accelerating expansion that doubled the Hubble parameter and hence doubled the size of the universe. The resulting accelerating expansion has provided the 90\% intergalactic baryons, and effectively the universe baryon average, with one extra bit/parameter to counteract the reduction of one bit/parameter in algorithmic information due to star formation (horizontal dashed line of Figure 3). Then accelerating expansion appears to be required to ensure the universe's algorithmic information complies with the $2^{\text {nd }}$ law, supporting our suggestion of a direct link between star formation, information and accelerating expansion.

\section{Information Energy also contributes to dark matter attributed effects.}

Although this work concentrates on assessing information energy as a source of dark energy that might account for Hubble Tension, we note that information energy probably also contributes to some effects previously attributed to dark matter. Space-time will be distorted equally by accumulations of matter and by accumulations of energy. While the above dark energy effects of information energy are effectively repulsive universe-wide, the extra distortions to space-time around structures due to information energy will be locally attractive and mimic dark matter. If information energy can account for dark energy then it must be strong enough to also produce significant dark matter like effects. By the nature of 
information energy these effects will be hard to quantify, so here we list below some qualitative effects that provide some circumstantial evidence.

A high correlation has been found [74,75] showing dark matter effects are fully specified by the baryons as would be expected if information energy contributes to those effects. Also the strongest dark matter effects in clusters of galaxies are found in the brightest and therefore highest temperature galaxies [76], again consistent with information energy being concentrated where baryons occur at high temperature and density. Clusters of colliding galaxies are considered to provide some of the strongest evidence for the existence of dark matter. Optical observations show stars pass through the collision largely unhindered whereas Xray observations show the galactic gas clouds, containing the majority of baryons, collide, slowing down or even halting. The location of dark matter is then identified from lensing measurements [77-79]. A study of the Bullet cluster [77], and of a further 72 mergers [78], both major and minor, finds no evidence for dark matter deceleration, with the dark mass remaining closely co-located with the stars and structure. Information energy could equally explain these effects, as information energy from stellar heated gas and dust passes along with the stars straight through the collision.

In our information energy explanation for dark energy, we require the present universe-wide information energy fraction of all energy, $f_{I E} \sim 68 \%$, to explain universe expansion history. Then, if information energy accounted for any significant fraction of dark matter attributed effects, we expect those effects to support a universe-wide dark matter fraction of all matter, $f_{D M} \sim 68 \%$. This value is significantly lower than $f_{D M} \sim 85 \%$ of the $\Lambda$ CDM model. A survey [80] of $1.7 \times 10^{5}$ massive early-type galaxies $\mathrm{z}<0.33$ yields $f_{D M}$ $=53 \%-72 \%$ within those galaxies' effective radius (radius defining the sphere responsible for $50 \%$ light emission). Another survey of 584 typical star-forming galaxies, $\mathrm{z}=0.8-1.0$ [81] finds $f_{D M}=65 \pm 12 \%$. Note the present expected information energy value $\sim 68 \%$ lies in the middle of both ranges but the $\Lambda \mathrm{CDM} f_{D M} \sim 85 \%$ lies outside.

While values of $f_{I E}$ should be high in each high temperature baryon object that we observe, universe average $f_{I E}$ would have been much lower at earlier times when only a small fraction of baryons had formed stars. Therefore, we expect observable object $f_{D M}$ values at or above the universe average $f_{I E}$ value for that redshift, with $f_{I E}$ effectively providing a 'minimum' value. In contrast, universe average $\Lambda \mathrm{CDM} f_{D M}$ should have remained constant, independent of redshift, and observed object $f_{D M}$ values should be found distributed approximately evenly about $f_{D M} \sim 85 \%$. Figure 4 compares these average and minimum values with several galaxy surveys [80-82] and with six early star forming disk galaxies [83].

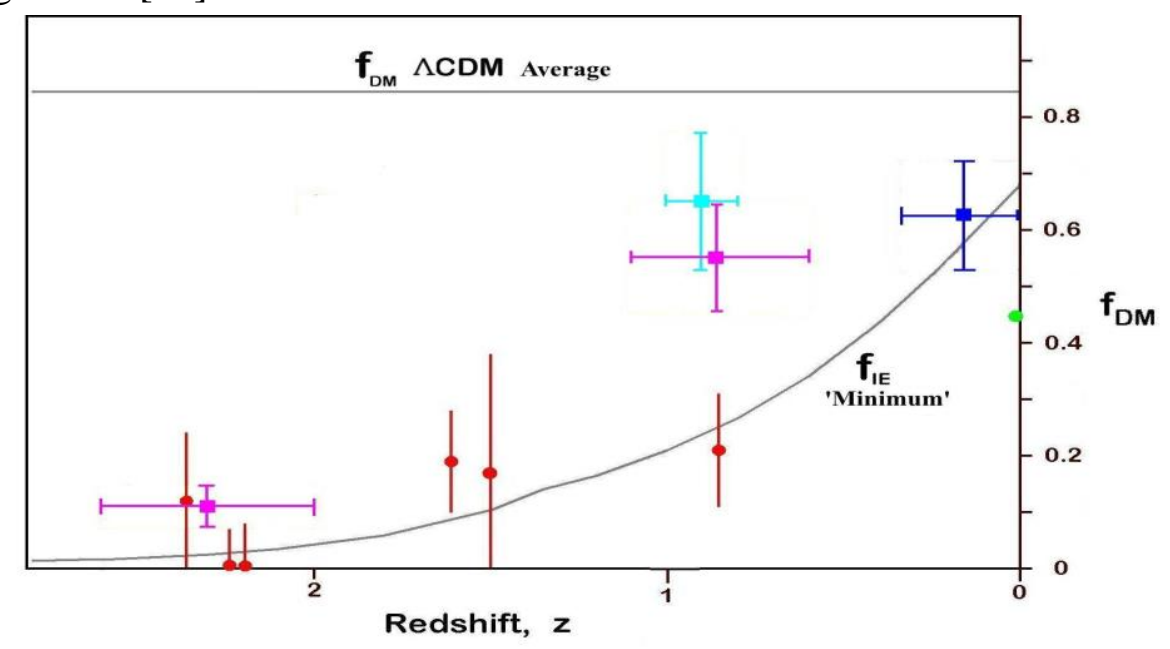


Figure 4. Observed $f_{D M}$ compared with average $\Lambda \mathrm{CDM} f_{D M}$ and the information energy $f_{I E}$. 'minimum'. Survey results, square symbols: Dark Blue, $1.7 \times 10^{5}$ massive early-type galaxies $\mathrm{z}<0.33$ [80], Light Blue, 584 typical star-forming galaxies, $\mathrm{z}=0.8-1.0$ [81], Purple, 92 star forming galaxies, $\mathrm{z}=2.0-2.6$ and 106 star forming galaxies, $\mathrm{z}=0.6-1.1$ [82]. Individual galaxies, circle symbols: Red, six early star forming galaxies [83]; Green, Milky Way galaxy [84]. The $f_{I E}$ curve is calculated from the power law fits to the star formation data of Fig.1, assuming present $f_{I E}=68 \%$.

The variation in the dark matter contribution illustrated in Figure 4 emphasizes the relative absence of dark matter effects in early massive star forming galaxies of 10 billion years ago. Surveys of early star forming galaxies made with the same instruments and analysis techniques, but in two different redshift ranges [82] (purple squares in Fig.4) show that galaxies at $\mathrm{z}=2.0-2.6$ clearly have lower $f_{D M}$ values than those at $\mathrm{z}=0.6-1.1$.

Overall, both survey and individually measured values of $f_{D M}$ are more consistent with the time varying minimum predicted by information energy than with the time invariant $85 \%$ average value of the $\Lambda \mathrm{CDM}$ model. In the $\Lambda \mathrm{CDM}$ model, clumps of dark matter attract baryons to form stars and structure. Over time, as more baryons join these clumps, we should expect a decrease of the observed dark matter fractions, contrary to the observations in Figure 4. In contrast, information energy causing dark matter-like effects could explain how the early massive galaxies with little dark matter, grew into the galaxies that we observe today, apparently dominated by dark matter, consistent with Figure 4.

The spatial distributions of some galaxies and galaxy clusters have been found to exhibit an "assembly bias" [85]. The way in which those galaxies interact with their dark matter environments appears to be determined not just by their mass but also by their past formation history. This could also be consistent with an information energy explanation since information/entropy results not just from present processes but depends on the past history of physical processes that operated on baryons.

The milky way, Andromeda, and Centaurus-A galaxies have a number of satellite dwarf galaxies that orbit in the same plane with the majority co-rotating [86]. This observation is difficult to reconcile with $\Lambda \mathrm{CDM}$ as dark matter should be distributed in a sphere around the parent galaxy with satellite galaxies randomly distributed. However, this observation may be consistent with information energy as dark matter-like effects should then follow the location of the parent galaxy's hot baryons.

\section{Summary}

We have shown that information energy must provide a significant source of dynamic dark energy. Overall, an information energy model would fit most data as well as the $\Lambda \mathrm{CDM}$ model, and at the same time resolve the Hubble tension problem. Furthermore, this model would solve the cosmological coincidence problem and allow the cosmological constant to take the more likely zero value. Information energy could also account for 
many effects previously attributed to dark matter. The information energy approach employed here emphasizes the two preferred requirements [87] of 'simplicity' (wielding Occam's razor) and 'naturalness' (relying on mostly proven physics), with a strong dependence on empirical data.

\section{Acknowledgments: The author is grateful for an Emeritus Professorship of Space} Science from the University of Sussex.

Funding: This research received no external funding.

Conflicts of Interest: The author declares no conflict of interest.

\section{References}

1. Planck Collaboration, Aghanim,N., Akrami, Y., Ashdown, A., et al., Planck 2018 results. VI, Cosmological Parameters, $A \& A$, 2020, 641, A6 (67 pp).

2. Choi, S.K., Hasselfield,M., Ho, S-Ppatty., et al., The Atacama Cosmology Telescope: A measurement of the cosmic microwave power spectra at 98 \& 150GHz, JCAP, 2020, 12, 045.

3. Abbott,T.M.C.,Abdalla,F.B., Annis,J., et al., Dark Energy Survey year 1 results: A precise H0 measurement from DES, Bao, D/H data. MNRAS, 2018, 480, 3879-3888.

4. Reiss A.G., Casertano,S., Yuan,W., et al., Large Magellanic Cloud Cepheid Standards provide a 1\% foundation for determination of $\mathrm{H} 0$ and strong evidence for physics beyond $\Lambda \mathrm{CDM}, A p$. J., 2019, 876, 85.

5. Reiss,A.G., Casertano,S., Yuan,W., et al., Cosmic distances calibrated to $1 \%$ precision with GAIA EDR3 parallaxes and Hubble Space Telescope photometry of 75 Milky Way Cepheids confirm tension with $\Lambda \mathrm{CDM}, 2020, \mathrm{ArXiV} 2012.08534 \mathrm{v} 1$.

6. Wong,K.C., Suyu,S,H., Chen,G.C.F., et al., HOLiCOW-XIII. A $2.4 \%$ measurement of H0 from lensed quasars: 5.3 tension between early- and late- Universe probes, MNRAS, 2020, 498,1420-1439.

7. Pesce,D.W., Braatz,J.A., Reid,M.J., et al., The megamaser Cosmology Project. XIII. Combined Hubble constant constraints, ApJL, 2020, 691, L1.

8. Freedman,W.L., Madore,B.F., Hatt,D., et al., The Carnegie-Chicago Hubble Program VIII: An independent determination of $\mathrm{H} 0$ based on the tip of the Red Giant Branch, ApJ, 2019, 882, 23.

9. Yuan,W., Reiss,A.G., Macri,L., et al., Consistent Calibration of the Tip of the Red Giant Branch in the Large Megallanic Cloud, ApJ, 2019, 886, 61.

10. Bernal, J.L., Peacock, J.A., Conservative Cosmology: combining data with allowances for unknown systematics, JCAP, 2018, 07, 002.

11. Verde,L., Treu,T., Riess,A.G., Kalvi Institute for Theoretical Physics Workshop Meeting Report: Tensions between the early and late Universe, Nat. Astron., 2019, 3, 891-895.

12. Landauer, R., Irreversibility and heat generation in the computing process. IBM J. Res. Dev. ,1961, 3, 183191.

13. Landauer, R., Information is physical. Phys. Today, 1991, 44, 23-29.

14. Toyabe,S., Sagawa,T., Ueda,M., et al., Experimental demonstration of information-to-energy conversion and validation of the generalized Jarzynski equality. Nat. Phys., 2010, 6, 988-992.

15. Berut, A., Arakelyan,A., Petrosyan, A., et al., Experimental verification of Landauer's principle linking information and thermodynamics. Nature, 2012, 483, 187-189.

16. Jun, Y., Gavrilov,M., Bechhoefer,J., High-Precision Test of Landauer's Principle in a Feedback Trap, Phys. Rev. Lett. 2014, 113, 190601-1 to -5.

17. Yan, L.L.,Xiong,T.P., Rehan,K., et al., Single_Atom Demonstration of the Quantum Landauer Principle, Phys. Rev. Lett. 2018, 120, 210601.

18. Frampton,P.H., Hsu,S.D.H., Kephart, T.W., Reeb,D., What is the entropy of the universe? Class. Quant. Grav., 2009, 26, 145005, (7pp).

19. Egan, C.A., Lineweaver, C.H.,A larger estimate of the entropy of the universe. ApJ., 2010, 710, 1825-1834.

20. Gough,M.P.,Holographic Dark Information Energy, Entropy, 2011, 13,924-935.

21. Gough,M.P., Holographic Dark Information Energy: Predicted Dark Energy Measurement, Entropy, 2013, 15, 1133-1149.

22. Gough,M.P., A Dynamic Dark Information Energy Consistent with Planck Data, Entropy, 2014, 16, 19021916.

23. Li, C., White, S.D.M., The distribution of stellar mass in the low-redshift universe, MNRAS, 2009, 39, 2177-2187. 
24. Gallazzi,A., Brinchmann, J., Charlot,S., White,S.D.M.,A census of metals and baryons in stars In the local universe, MNRAS, 2008, 383, 1439-1458.

25. Moustakas, J., Coil,A., Aird, J., et al., PRIMUS: constraints on star formation quenching and Galaxy merging and the evolution of the stellar mass function from z=0-1, ApJ., 2013, 767, 50 (34pp).

26. Bielby,R., Hudelot,P., McCracken,H.J., et al., The WIRCam Deep Survey. I. Counts, colours, and mass functions derived from near-infrared imaging in the CFHTLS deep fields, $A \& A, \mathbf{2 0 1 2}, 545$, A23 (20pp).

27. Perez-Gonzalez,P.G.,Rieke, G.H., Villar V, et al., The stellar mass assembly of galaxies from z=0-4: analysis of a sample selected in the rest-frame near infrared with Spitzer, ApJ., 2008, 675, 234-261.

28. Ilbert, O., McCracken, H.J., Le F`evre, O., et al., Mass assembly in quiescent and star-forming Galaxies since $\mathrm{z} 44$ from UltraVISTA, $A \& A, \mathbf{2 0 1 3}, 556$, A55 (19pp).

29. Muzzin, A., Marchesini,D., Stefanon, M., et al. The evolution of the stellar mass functions of star-forming and quiescent galaxies to $\mathrm{z}=4$ from the COSMOS/UltraVISTA survey, ApJ., 2013, 777, 18 (30pp).

30. Arnouts, S., Walcher, C.J., Le Fevre, O., et al.,The SWIRE-VVDS-CFHTLS surveys: Stellar Assembly over the last 10Gyr., A\&A, 2007, 476, 137-150.

31. Pozzetti, L., Bolzonella, M., Zucca,E.,et al., zCOSMOS -10k bright spectroscopic sample.The bimodality in thegalaxy stellar mass function, $A \& A, \mathbf{2 0 1 0}, 523, \mathrm{~A} 13$ (23pp).

32. Kajisawa, M., Ichikawa, I. , Tanaka, I., et al. MOIRCS deep survey IV evolution of galaxy stellar mass function back to z 3, Ap. J., 2009, 702, 1393-1412.

33. Marchesini, D., van Dokkum,P.G., Forster Schreiber, N.M., et al., The evolution of the stellar mass function of galaxies from $\mathrm{z}=4$ and the first comprehensive analysis of its uncertainties, Ap. J., 2009, 701, 1765-1769.

34. Reddy, N.A., Dickinson, M., Elbaz, D., et al. GOODS-HERSCHEL measurements of the dust attenuation of typical star forming galaxies at high redshift, Ap. J., 2012, 744, 154(17pp).

35. Caputi, K.I., Cirasuolo, M., Dunlop, J.S., et al. The stellar mass function of the most massive Galaxies at $3<\mathrm{z}<5$ in the UKIDSS Ultra Deep Survey, MNRAS, 2011, 413, 162-176.

36. Gonzalez,V., Labbe, I., Bouwens, R.J., et al. , Evolution of galaxy stellar mass functions, mass densities, and mass-to light ratios from z 7 to z 4, Ap. J. Let. , 2011, 735, L34(6pp).

37. Lee,K.S., Ferguson,H.C., Wiklind,T., et al., How do star-forming galaxies at $\mathrm{z}>3$ assemble their masses?, Ap.J., 2012, 752, 66(21pp).

38. Yabe, K., Ohta, K., Iwata, I., et al. The stellar populations of Lyman break galaxies at z 5, Ap. J. 2009, 693, 507533.

39. Labbe,I., Oesch, P.A., Bouwens,R.J., et al., The spectral energy distributions of $\mathrm{z} \sim 8$ galaxies from the IRAC ultra deep fields, Ap. J. Let. 2013, 777, L19(6pp).

40. Madau, P., Dickinson, M., Cosmic Star Formation History, Ann. Rev. Astron. and Astrophys. 2014, 52, 415-486.

41. Cole,S., Peterson, B.A., Jackson, C., et al., The 2dF galaxy redshift survey, MNRAS, 2001, 326, 255-273.

42. Dickinson, M., Papovich, C., Ferguson,H.C., et al., The evolution of the global stellar mass density a0 $<\mathrm{z}<3$. ApJ. 2003, 587, 25-40.

43. Rudnick,G., Rix,H.W., Franx,M., et al., The rest-frame optical luminosity density, colour, and stellar mass density of the universe from $\mathrm{z}=0$ to $\mathrm{z}=3$., ApJ., 2003, 599, 847-864.

44. Brinchmann, J., Ellis, R.S.,The mass assembly and star formation characteristics of field galaxies of known morphology, ApJ. , 2000, 536, L77-L80.

45. Elsner, F., Feulner, G., Hopp,U., The impact of Spitzer infrared data on stellar mass estimates. A\&A, 2008, 477, 503-512.

46. Drory, N., Salvato, M., Gabasch, A., et al, The stellar mass function of galxies to z 5, ApJ., 2005, 619, L131-L134.

47. Drory, N., Alvarez,M., The contribution of star formation and merging to stellar mass buildup in galaxies, ApJ, 2008, 680, 41-53.

48. Fontana, A., et. al., The assembly of massive galaxies from near Infrared observations of Hubble deep-field south, Ap. J. Lett. 2003, 594, L9-L12.

49. Fontana, A., et al., The galaxy mass function up to $\mathrm{z}=4$ in the GOODS-MUSIC sample, $A \& A, \mathbf{2 0 0 6}, 459,745-757$.

50. Cohen, J.G., CALTECH faint galaxy redshift survey. Ap.J. , 2002, 567, 672-701.

51. Conselice, C.J., Blackburne, J.A., Papovich, C., The luminosity, stellar mass, and number density evolution of field galaxies. Ap.J. .2005, 620, 564-583.

52. Borch,A., Meisenheimer, K., Bell, E.F., et al.,The stellar masses of 25000 galaxies at $0.2<\mathrm{z}<1.0$ estimated by COMBO-17 survey, $A \& A, \mathbf{2 0 0 6}, 453,869-881$.

53. Chiang, Y-K, Makiya, R., Ménard, B., et al. The Cosmic Thermal History Probed by Sunyaev-Zeldovich Effect Tomography , Ap.J, 2020, 902, 56.

54. T'Hooft,G., Obstacles on the way towards the quantization of space, time and matter- and possible solutions, Stud. Hist. Phil. Mod. Phys. 2001, 32, 157-180. 
55. Susskind, L., The world as a hologram. J. Math. Phys., 1995, 36, 6377-6396.

56. Buosso,R., The holographic principle. Rev. Mod. Phys. , 2002, 74, 825-874.

57. Weinberg, A., Gravitation and Cosmology, Wiley, New York, 1972.

58. Friedman, A., On the Curvature of Space, General Relativity and Gravitation, 1999, 31-12, 1991-2000.

59. ESA Euclid Science Requirements Document https://sci.esa.int/documents/33220/36137/1567257215944Euclid_SciRD_DEM-SA-DC-0001_4_0_2010-03-22.pdf. (Accessed 24 April 2020)

60. Ade, P., et al., Plank Collaboration. Planck 2013 results. XVI. Cosmological parameters, A\&A, 2014, 571, A16.

61. Ade, P., et al., Plank Collaboration. Planck 2015 results. XIII. Cosmological parameters, A\&A, 2016, 594, A13.

62. Ade, P. et al., Planck Collaboration. Planck 2015 results. XIV. Dark energy and modified gravity, A\&A, 2016, 594, A14.

63. Chevallier, M., Polarski, D., Accelerating universes with scaling dark matter. Int. J. Mod. Phys.D., 2001,10, 213-224.

64. Rest, A., Scolnic,D., Foley, R.J., et al., Cosmological constraints from measurements of type 1A supernovae discovered during the first 1.5 years of the Pan_STARRs1 survey, ApJ. , 2014, 795, 44(34pp).

65. Shafer, D.L., Huterer, D., Chasing the phantom: A closer look at type 1A supernovae and the dark energy equation of state, Phys. Rev. D, 2014, 89, 063510(11pp).

66. Cheng, C., Huang,Q-G., Dark side of the universe after Planck data, Phys. Rev. D, 2014, 89, 043003, (8 pp).

67. Xia, J-Q., Li, H., Zhang, X., Dark energy constraints after new Planck data, Phys. Rev. D, 2013, 88, $063501,8 \mathrm{p}$

68. Delubac, T., Bautista, J.E., Busca, N.G., et al., Baryon acoustic oscillations in the Lya forest of BOSS DR11 quasars, $A \& A, \mathbf{2 0 1 5}, 574$, A59 (17pp).

69. Frieman, J.A., Turner, M.S., Huterer, D., Dark energy and the accelerating universe, Ann.Rev.Astron. Astrophys. 2008, 46, 385-432.

70. Guzzo, L., Pierleoni, M., Meneux, B., et al., A test of the nature of cosmic acceleration using galaxy redshift distortions. Nature, 2008, 451, 541-544.

71. Weinberg, S., The cosmological constant problem. Rev. Mod. Phys. 1989, 61, 1-23.

72. Migkas, K., Schellenberger, G., Reiprich, T.H., et. al., Probing cosmic isotropy with a new X-ray galaxy cluster sample through the $\mathrm{L}_{\mathrm{x}}-\mathrm{T}$ scaling relation, $A \& A, \mathbf{2 0 2 0}, 636$, A15.

73. Devine, S., The Insights of Algorithmic Entropy, Entropy, 2009, 11, 85-110.

74. McGaugh, S.S., Lelli, F., Schomobert, J.M., The Radial Acceleration Relation in Rotationally Supported Galaxies, Phys. Rev. Lett., 2016, 117, 201101.

75. Lellil, F., McGaugh, S.S., Schombert, J.M., Pawlowskil, M.S., One Law to Rule them all: the Radial Acceleration relation of Galaxies, ApJ. , 2017, 836, 152 (23pp).

76. Viola, M., Cacciato,M., Brouwer, M., et al., Dark matter halo properties of GAMA galaxy groups from 100 square degrees of KiDS weak lensing data, MNRAS, 2015, 452, 3529-3550.

77. Markevich, M., Gonzalez, H., Clowe, D.,A., et al., Direct constraints on the dark matter self-interaction crosssection from the merging galaxy cluster 1E0657-56, ApJ. , 2004, 606-2, 819-824.

78. Harvey, D., Massey, R., Kitching, T., et al., The non gravitational interactions of dark matter in colliding galaxy clusters, Science, 2015, 347 - 62290, 1462-1465.

79. Massey, R., Williams, L., Smit, R., et al., The behaviour of dark matter associated with four bright Cluster galaxies in the 10kpc core of Abell 3827. MNRAS, 2015, 449(4), 3393-3406.

80. Grillo, C., Projected central dark matter fractions and densities in massive early-type galaxies from the Sloan Digital Sky Survey, ApJ., 2010, 722, 779-787.

81. Stott,J.P.,Swinbank,A.M., Johnson,H.L.,et al.,The KMOS Redshift One Spectroscopic Survey (KROSS): Dynamical properties, gas and dark matter fractions of typical $\mathrm{z} \sim 1$ star-forming Galaxies, MNRAS, 2016, 457, 1888-1904.

82. Wuyts, S., et al. KMOS3D: Dynamical constraints on the mass budget in early star-forming disks. ApJ., 2016, 831, 149-171.

83. Genzel, R., Forster N.M., Schreiber, et al., Strongly baryon-dominated disk galaxies at the peak of galaxy formation ten billion years ago, Nature, 2017, 543, 397-401.

84. Bland-Hawthorn, J., Gerhard, O., The galaxy in context: structural, kinematic, and integrated properties. Ann. Rev. Astron. Astrophys., 2016, 54, 529-596.

85. Miyatake, H., More, S., Takada, M., Spergel,D.N.,et el., Evidence of Halo Assembly Bias in Massive Clusters. Phys. Rev. Lett. 2016, 116, 041301 (5pp).

86. Muller, O., Pawlowski, M.S., Jerjen, H., Lelli, F., A whirling plane of satellite galaxies around Centaurus A challenges CDM cosmology, Science, 2018, 359, 534-537. 
87. Bull, P., Akrami, Y., Adamek, J., et al., Beyond $\Lambda$ CDM: Problems, solutions, and the road ahead, Physics of the Dark Universe, 2016, 12, 56-99. 